\title{
Comparative study of histological changes (thermal artefacts) in resected specimens of monopolar and bipolar trans-urethral resection of bladder tumours
}

\author{
Vasudevan Thirugnanasambandam ${ }^{1}$, Jeyaraman Ramanathan ${ }^{2}$
}

Cite this article: Thirugnanasambandam $\mathrm{V}$, Ramanathan J: Comparative study of histological changes (thermal artefacts) in resected specimens of monopolar and bipolar trans-urethral resection of bladder tumours. Ann Urol Oncol 2020; 3(2): 82-88. https:// doi.org/10.32948/auo.2020.10.30

\begin{abstract}
Objective The primary aim of the study was to study the histological changes (Thermal artefacts) noted in the resected specimens between the Monopolar and Bipolar Trans-Urethral Resection of Bladder Tumours (TURBT).

Patients and Methods The study was done between March 2012 and Feb 2013. This was a comparative study between Monopolar and Bipolar resection specimens studied for histological changes (Thermal artefacts). Institutional Ethics Committee approval was obtained. Informed consent was taken from all patients. Patients were randomized into two groups, Monopolar Group or Bipolar Group of 50 each to undergo TURBT. Patients either underwent Monopolar or Bipolar TURBT in Glycine and Saline respectively.

Results Thermal artefacts were graded according to WHO (World Health Organization) grading system. There was no difficulty noticed during histo-pathological examination of resected specimens. In Monopolar group, there were 27 (54\%) grade 2 thermal damage, $17(34 \%)$ grade 1 thermal damage and no grade-3 thermal damage in examined specimen. In Bipolar group, there were 07 (14\%) grade 2 thermal damage, 12 (24\%) grade 1 thermal damage and no grade 3 thermal damage in examined specimen.

Conclusion The degree of thermal damage is much lesser in histological sections of specimen resected using Bipolar energy and interpretation of the grade is easier which is one of the most important prognosticators especially in bladder tumours since high grade lesions are proven beyond doubt to progress and recur.
\end{abstract}

Key words Bipolar, monopolar, turbt, thermal artefacts, electrosurgery

\footnotetext{
1. Department Of Urology, Mahatma Gandhi Medical College And Research Institute, Pondicherry, India.

2. Department Of Urology, Sri Balaji Medical College, Chennai, India.

Correspondence: Vasudevan Thirugnanasambandam (Department Of Urology, Mahatma Gandhi Medical College And Research Institute, Sri Balaji Vidyapeeth, Pondicherry-607402; E-mail: akashlaya@gmail.com).
} 


\section{Introduction}

Bladder malignancy is the fourth and eighth common tumour in men and women respectively. Also it is the 9th most common cancer worldwide [1]. In 1910 Beer was the first to do an endoscopic resection of bladder tumour. Since then TURBT has remained the basic diagnostic and often therapeutic tool for Bladder tumours [1].

Histologically, Urothelial carcinomas constitute about $90 \%$ of bladder cancers, remainder $5 \%$ by Squamous cell carcinomas, and less than $2 \%$ by Adenocarcinoma. Overall Urothelial carcinoma is the commonest cancer of the urinary tract and stands second in position next only to renal cell carcinoma leading to death in genitourinary tumors. About $80 \%$ of Urothelial tumors are diagnosed early in patients when still the tumour is superficial and non-muscle invasive [2].

The primary treatment for visible lesion is transurethral resection of the bladder tumor (TURBT) under anesthesia with the aim to (1) resect all visible growth and (2) obtain adequate specimens for histopathological grading and staging.

The technique of TURBT is well known and the difference between Monopolar and Bipolar lies with the path of the current circuit.

In Monopolar electrosurgery the path of the electric current is from the active loop, through the tissues (patient), through the indifferent electrode placed abutting the patient's skin and back to the electro-surgical unit to complete the circuit. The heat generated at the loop-tissue interface is used for resection.

In Bipolar electrosurgery, the large return electrode of the Monopolar mode is replaced with a second small electrode. The path of the electric current is from the active loop, through the conducting irrigant, through the patient's tissue, to the second indifferent electrode which is placed very close within the same loop and then back to the electrosurgical generator. Two electrodes are combined in the instrument. Current passes between tips and not through the patient. Hence, there is no current flowing through the patient's body.

In monopolar systems, since the electrical energy is carried through the tissue, resistance offered by the tissue elevates temperature to as high as $400 \mathrm{oC}$ which leads to tissue damage and desiccation making difficulty in interpreting pathological specimens $[3,4]$.

But in bipolar energy, the radiofrequency current converts the conducting irrigant into plasma which is at a higher temperature which dissociates the bonds between the tissues reducing it into elementary molecules.

Since the current is not completely running through the tissues and is circuited through the closely placed indifferent electrode temperature rise in the tissues resected is only modest $(40$ to $70 \mathrm{oC}$ ) than during monopolar surgery and the collateral spread is less [3, 4].

With this background, a study was conducted to know the histological changes (Thermal artefacts) between Monopolar and Bipolar energy.

\section{Materials and Methods}

\section{Period of study}

The study was done between March 2012 and Feb 2013 in a tertiary care centre.

Type

This was a comparative study evaluating the histological changes (Thermal artefacts) in the resected specimens of Monopolar and
Bipolar Trans-Urethral Resection of Bladder Tumours (TURBT).

\section{Inclusion criteria}

All patients diagnosed to have Bladder tumour by Ultrasonography, Contrast Enhanced Computed Tomography (CECT) and Cystoscopy.

\section{Exclusion criteria}

Patients with coagulopathy (INR $>2$ ).

Seriously ill patients ( Karnofsky score $<50$ ).

\section{Method of Study}

Institutional Ethics Committee approval was obtained. Informed consent was taken from all patients. Patients were randomized into two groups of 50 each to undergo TURBT either Monopolar Group or Bipolar Group based on random numbers generated by computer software and each number placed inside a closed envelope and on the day of surgery in the morning where in an operation staff assistant was asked to pick the envelope and patient allotted accordingly. Patients either underwent Monopolar or Bipolar TURBT in Glycine and Saline respectively.

We used the Gyrus ACMI Plasma-Kinetic (PK) system and used PK thin loop for resection. The settings we employed were $160 \mathrm{~W}$ cutting and 80W coagulation respectively for Bipolar resection.

Martin ME MB2 monopolar system was used for Monopolar resection at the setting of $120 \mathrm{~W}$ cutting and $60 \mathrm{~W}$ coagulation.

\section{Procedure}

All the resections were performed by a single surgeon who was very experienced in performing TURBT. A 26-F Karl Storz continuous flow resectoscope was used with either glycine or saline irrigation for Monopolar TURBT and for Bipolar TURBT.

Tumour resection was done in a step by step manner starting from the summit of the tumour so as to de-florate reaching the stalk or the base. Once the base was resected, the underlying muscle was resected separately. The two specimens superficial and deep were put in separate containers and sent for histo-pathological examination. The specimens sent to Pathology was examined and reported by the Head of the department, Pathology. Moreover the pathologist was blinded to the technique of surgery whether Monopolar or Bipolar.

Thermal damage produced by the electrosurgery has been graded using the WHO Grading [5, 6] system (Table 1). It has been graded into four grades according to the difficulty in identifying the cellular architecture and as the grade increases the more is the difficulty in identifying the tissue architecture.

\section{Observation and Results}

Pre-operative parameters of both Group 1 and Group 2 are presented in Table 2.

\section{Monopolar Group}

The Histopathological examination of the tissue specimens yielded a diagnosis of Transitional Cell Carcinoma in 48 patients. Out of this 48 transitional cell carcinoma, Papillary histology was found in 17, Low-grade T1 in 16, High-grade T1 in 12 and Muscle invasive in 3 specimens. The histopathology of two specimens turned out to be Adenocarcinoma and Squamous Cell Carcinoma one in each respectively. All the specimens were examined and reported without any difficulty. 
Table 1. World Health Organization (WHO) thermal grade.

\begin{tabular}{ll} 
Degree of thermal damage & Characterization \\
\hline 0 & $\begin{array}{l}\text { No thermal damage } \\
\text { Lowest grade of thermal artifacts. The cellular structure is } \\
\text { identifiable and not impaired } \\
\text { Medium grade. Cellular structure and nuclei are impaired, but still } \\
1\end{array}$ \\
$\begin{array}{l}\text { identifiable } \\
\text { High grade artifacts. Complete loss of the cellular structure. } \\
\text { No differentiation of the cellular parts }\end{array}$ \\
\hline
\end{tabular}

When the thermal artefact grade was analysed the mean grade was 1.42 (Figure 1).

Bipolar Group

The Histopathological examination of the tissue specimen yielded a diagnosis of Transitional Cell Carcinoma in 49 patients. Out of this 49 transitional cell carcinoma Papillary histology was found in 23, Low-grade T1 in 16, High-grade T1 in 08 and Muscle invasive in 2 specimens. The histopathology of one specimen turned out to be Squamous Cell Carcinoma. All the specimens were examined and reported without any difficulty.

When we analysed the thermal artefacts the mean grade approached 0.54 (Figure 1).

Thermal Artefact

Thermal artefacts were graded according to WHO grading

Table 2. Comparative patient and tumour demographics of monopolar and bipolar group.

\begin{tabular}{llll}
\hline Variable & Monopolar & Bipolar & P Value \\
Number of patients(n) & 50 & 50 & \\
Male & 45 & 44 & 0.538 \\
Female & 5 & 6 & \\
Mean age (years) & 58.2 & 56.5 & 0.474 \\
Smoking(n) & 37 & 35 & 0.656 \\
Diabetes(n) & 14 & 15 & 0.834 \\
Hypertension(n) & 17 & 18 & 0.826 \\
Multiplicity(n) & & & \\
Single & 46 & 45 & 0.532 \\
Multiple & 4 & 5 & \\
Location(n) & & & \\
Lateral wall & 29 & 39 & \\
Posterior wall & 2 & 3 & \\
Anterior wall & 1 & 2 & \\
Trigone & 14 & 10 & \\
Dome & 7 & 4 & \\
\hline
\end{tabular}

$\mathrm{N}$ : Number. 


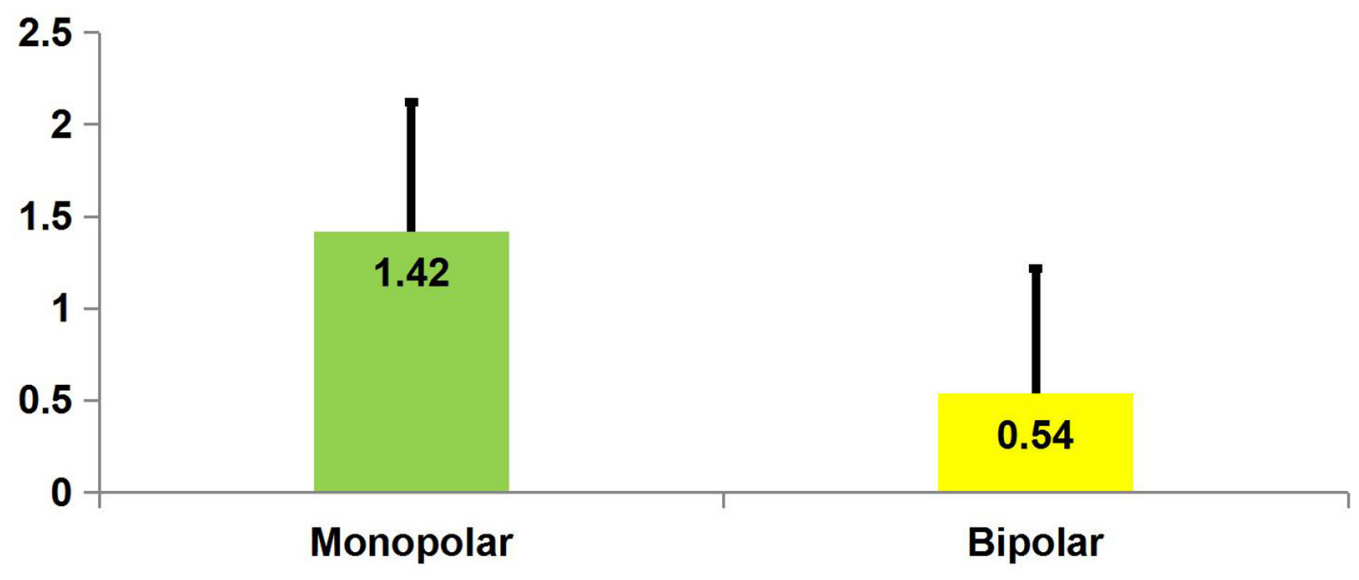

Group

Figure 1. Thermal artefact comparison in Monopolar and Bipolar groups.

system . There was no difficulty noticed during histopathological examination of resected specimens.

In Monopolar group, there were 27 (54\%) grade 2 thermal damage, 17 (34\%) grade 1 thermal damage and no grade- 3 thermal damage in examined specimen.

In Bipolar group, there were 07 (14\%) grade 2 thermal damage, $12(24 \%)$ grade 1 thermal damage and no grade 3 thermal damage in examined specimen (Figure 2).

Comparative Statistical analysis of Monopolar \& Bipolar group

The comparison of mean values of continuous and categorical variables was done using the Student's t-test and the chi-square test respectively. If the $\mathrm{p}$ value is $<0.05$, the result is considered statistically significant.

When analysing the thermal damage it was seen that there were more artefacts in Monopolar group (Table 3).

\section{Discussion}

Bladder malignancy is the most common tumour identified in patients more than $50 \mathrm{yrs}$ with hematuria [7]. Mean age of presentation is 70yrs in both the sexes and there is a progressive increase in the incidence and death with advancing age [8].
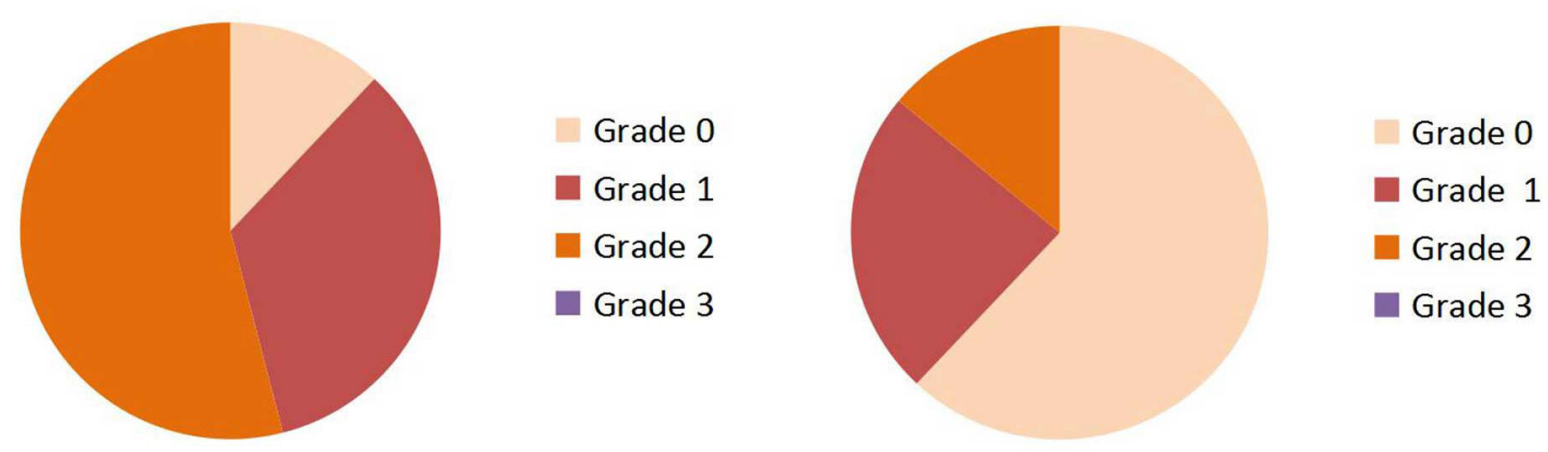

A

B

Figure 2. World Health Organization (WHO) thermal damage grading comparison between Monopolar(A) and Bipolar(B) TURBT. 
Table 3. Comparative statistics of Monopolar and Bipolar groups with regards to thermal artefact grade.

\begin{tabular}{llllll}
\hline Variable & Group & N & Mean & Standard Deviation & P-Values \\
\hline Thermal artefact grade & Monopolar & 50 & 1.42 & 0.70 & 0.001 \\
& Bipolar & 50 & 0.54 & 0.68 & \\
\hline
\end{tabular}

Overall Urothelial carcinoma is the commonest cancer of the urinary tract. About $80 \%$ of Urothelial tumors are diagnosed early in patients when still the tumour is superficial and non-muscle invasive [5].

After its introduction by Romanians, Bipolar resection technique is well established $[9,10]$.

The depth of penetration is more in monopolar resection when compared to bipolar resection (Figure 3). Hence some studies have quoted that bladder perforation being less in Bipolar when compared to Monopolar [11, 12]. One of the quoted advantages in literature is that, there is lesser degree of carbonisation and darkening while resecting bladder tumours employing Bipolar energy which allows excellent visualization of the residual tumour tissue after resection allowing complete resection [13].

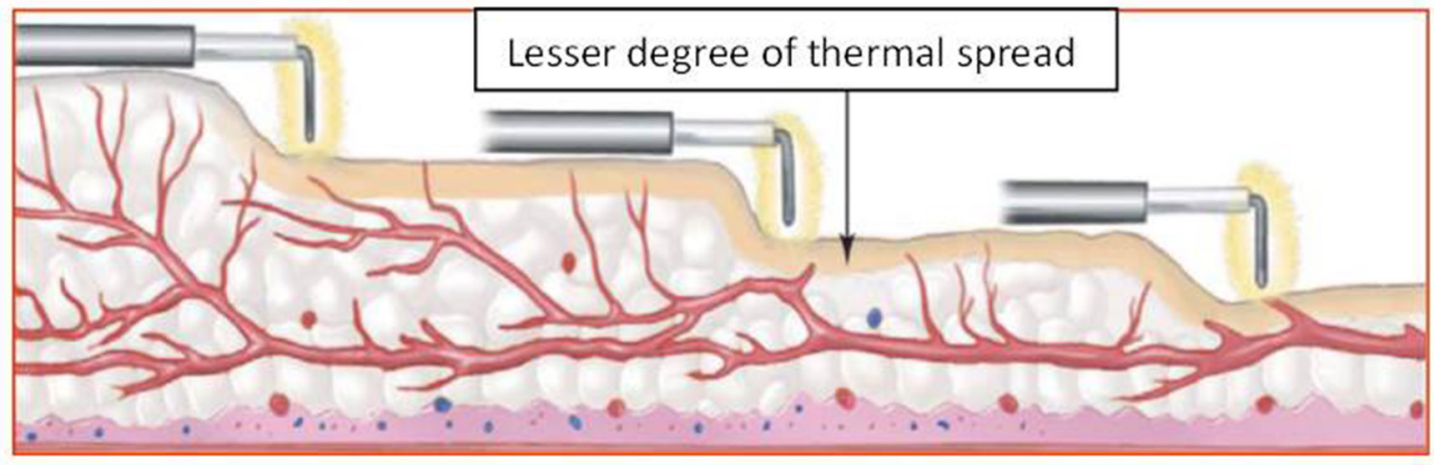

A

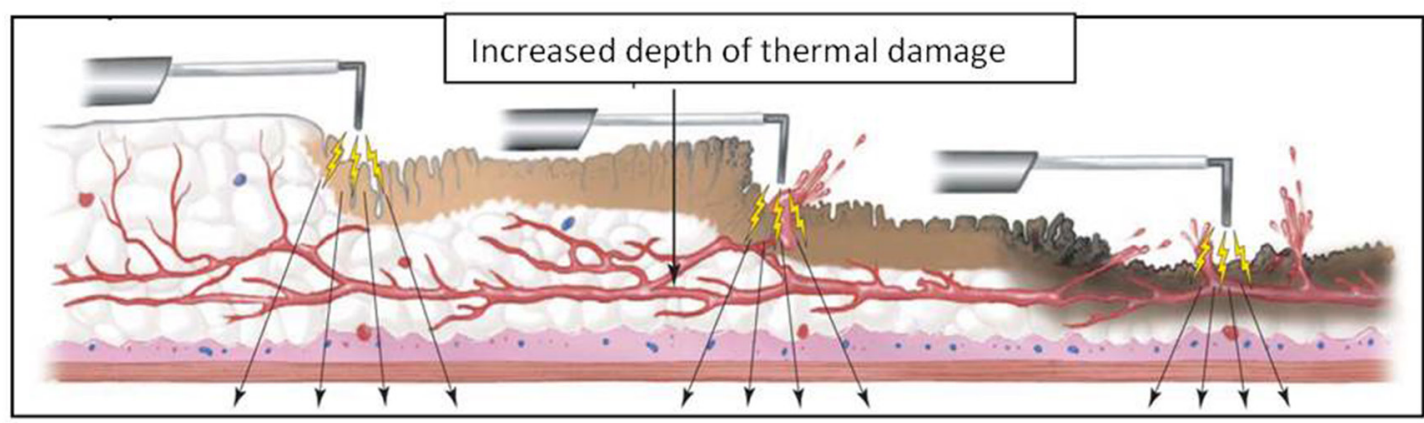

B

Figure 3. Thermal spread and its depth in Bipolar(A) and Monopolar(B) Electrosurgery. 
The specimen after resection from Bipolar TURBT is comparable from a histological point of view to that obtained using Monopolar TURBT, with few advantages of the Bipolar resection specimens showing lesser thermal damage.

Yang SJ et al have done a study to assess the deep tissue damage comparing Monopolar and Bipolar TURBT and did not find any difficulty in histopathological diagnosis between both energy sources [8].

Another study conducted by Meneghini A et al [14] noted that in Bipolar resections there were no thermal artefacts in resected specimens which substantially improved pathological interpretation through better evaluation of depth of muscle penetration, angiolymphatic invasion and adjacent mucosa.

Bach $\mathrm{T}$ et al in their study on Bipolar resection showed a decrease in resection time, decreased carbonisation of tissues and nonstickiness of the tissues to the loops [15].

With the advent and availability of bipolar energy source, many studies have been conducted and shown that even though the thermal damage produced by monopolar cautery is severe there was no difficulty in pathological interpretation [16, 17, 18].

The presence of deep muscle and its pathological interpretation carries a pivotal role in further management of bladder tumours. Some randomised studies have shown that deep muscle was present in $90-100 \%$ of monopolar specimen and $100 \%$ of bipolar specimens $[19,20,21]$. But in one of the studies the thickness of deep muscle was thinner when compared to monopolar [8].

When we examined our specimens, there was increased number and degree of artefacts noticed in Monopolar resections than bipolar resections when graded using WHO grading. But grade-3 thermal artefact was not noticed in both Monopolar TURBT and Bipolar TURBT.

All the artefacts noticed in the Bipolar TURBT were grade-1 and most of the Monopolar TURBT specimens showed grade-2 artefacts. On statistical analysis it was shown that Bipolar is superior in preserving the cytoarchitexture $(\mathrm{p}=0.001)$.

We have included both non-muscle invasive and muscle invasive tumours in our study because some cases of muscle invasive bladder tumours which were resected previously by monopolar energy were found difficult by the pathologists to interpret because of carbonisation.

In the present study, our pathologist was able to clearly identify invasion of muscle in Bipolar group without any difficulty. But in monopolar group even though invasion into muscle was identified it needed repeated sections of blocks of tissue before the results were announced.

Another thing in our study is we not only interpreted transitional cell carcinoma but we do found squamous and adenocarcinoma also. We aimed at identifying only the thermal artefacts and its impact on histological interpretation not at the type of histology since the damage produced by the electrocurrent is the same.

The limitation of our study is that a single pathologist was involved. It would have been better if the slides were reviewed by another peer pathologist to eliminate bias. But our pathologist was a senior person with good experience.

\section{Conclusion}

The conclusions that can be drawn from our present study is that the Bipolar resection when compared to Monopolar resection produces lesser degree of thermal artefacts, preservation of cytoarchitexture and thereby helping in correct interpretation of grade and depth of invasion. It was easy to identify muscle invasion without the need for repeat histological sections of bladder tumours resected by Bipolar energy.

\section{Ethical policy}

Approval was taken from institutional ethical committee. The study was performed in accordance with the Declaration of Helsinki. Patients gave their informed consent for their participation.

\section{Author contributions}

VT: Study design, data collection, manuscript editing; JR: Study protocol, supervision, manuscript review.

\section{Competing interests}

The authors declare no competing interests.

\section{Funding}

This research did not receive any specific grant from funding agencies in the public, commercial, or not-for-profit sectors.

\section{References}

1. Jemal A, Murray T, Ward E, Samuels A, Tiwari RC, Ghafoor A, et al: Cancer statistics, 2005. CA Cancer J Clin 2005; 55: 10-30.

2. Lopez-Beltran A: Bladder cancer: clinical and pathological profile. Scand J Urol Nephrol Suppl 2008; 218: 95-109.

3. Singh H, Desai MR, Shrivastav P, Vani K: Bipolar versus monopolar transurethral resection of prostate: randomized controlled study. J Endourol 2005; 19: 333-338.

4. Faul P, Schlenker B, Gratzke C, Stief CG, Reich O, Hahn RG: Clinical and technical aspects of bipolar transurethral prostate resection. Scand J Urol Nephrol 2008; 42: 318-323.

5. Wang DS, Bird VG, Leonard VY, Plumb SJ, Konety B, Williams $\mathrm{RD}$, et al: Use of bipolar energy for transurethral resection of bladder tumors: pathologic considerations. J Endourol 2004; 18: 578-582.

6. Lagerveld BW, Koot RA, Smits GA: Thermal artifacts in bladder tumors following loop endoresection: electrovaporization $\mathrm{V}$ electrocauterization. J Endourol 2004; 18: 583-586.

7. Thomas K, O'Brien T: Improving Transurethral Resection of Bladder Tumour:The Gold Standard for Diagnosis and Treatment of Bladder Tumours. Eur Urol 2008; 7 (Suppl): 524-528.

8. Yang SJ, Song PH, Kim HT: Comparison of Deep Biopsy Tissue Damage from Transurethral Resection of Bladder Tumors between Bipolar and monopolar Devices. Korean J Urol 2011; 52: 379-383.

9. Mahmoud MA, Tawfick A, Mostafa DE et al: Can bipolar energy serve as an alternative to monopolar energy in the management of large bladder tumours $>3 \mathrm{~cm}$ ? A prospective randomised study. Arab Journal of Urology. 2019; 17(2): 125-131.

10. Geavlete B, Multescu R, Georgescu D, Jecu M, Dragutescu M, Geavlete P: Innovative technique in nonmuscle invasive bladder cancer-bipolar plasma vaporization. Urology 2011; 77: 849-854.

11. Del Rosso A, Pace G, Masciovecchio S, Saldutto P, Galatioto GP, Vicentini C: Plasmakinetic bipolar versus monopolar transurethral resection of non-muscle invasive bladder cancer: a single center randomized controlled trial. Int J Urol 2013; 20: 399-403.

12. Mansour AM, Shokeir AA, Tharwat M, Ali-El-Dein B, Osman Y: Pd17-10 monopolar versus bipolar transurethral resection of non-muscle invasive bladder cancer: a single center randomized controlled trial. J Urol 2015; 193: 384-385.

13. Gupta NP, Saini AK, Dogra PN et al: Bipolar energy for transurethral resection of bladder tumours at low-power settings:initial experience. BJU 2010; 108: 553-556.

14. Meneghini A, Borghi L, Ballotta M.R et al: Bipolar transurethral resection of bladder cancer: pathomorphologic advantages Eur Urol Suppl 2007; 6(2): 173.

15. Bach T, Herrmann T, Cellarius $\mathrm{C}$ et al: Bipolar resection of the 
bladder and prostate - Initial experience with a newly developed regular sized loop resectoscope. J Med Life 2009; 2(4): 443-446.

16. Saini AK, Ahuja S, Seth A et al: Histomorphological features of resected bladder tumors: Do energy source makes any difference. Urol Ann 2015; 7(4): 466-469.

17. Huang X, Wang XH, Qu LJ et al: Bipolar Versus Monopolar Transurethral Resection of Prostate: Pathologic Study in Canines. Basic science 2007; 70(1): 180-184.

18. Murugavaithianathan P, Devana SK, Mavuduru R et al: Bipolar Transurethral Resection of Bladder Tumor Provides Better Tissue for Histopathology But Has No Superior Efficacy and Safety: A Randomized Controlled Trial. J Endourol 2018; 32(12): 1125-1130.

19. Osman, Y., Harraz, A.M: A Review Comparing Experience and Results with Bipolar Versus Monopolar Resection for Treatment of Bladder Tumors. Curr Urol Rep 2016; 17(21).

20. Mashni J, Godoy G, Haarer C, Dalbagni G, Reuter VE, Al-Ahmadie $\mathrm{H}$, et al: Prospective evaluation of plasma kinetic bipolar resection of bladder cancer: comparison to monopolar resection and pathologic findings. Int Urol Nephrol 2014; 46: 1699-705.

21. Venkatramani V, Panda A, Manojkumar R, Kekre NS: Monopolar versus bipolar transurethral resection of bladder tumors: a single center, parallel arm, randomized, controlled trial. J Urol 2014; 191: 1703-7. 